\title{
Utility of Lung Perfusion SPECT/CT in Detection of Pulmonary Thromboembolic Disease: Outcome Analysis
}

\author{
Teik Hin $\operatorname{Tan}^{1}$ (D) $\cdot$ Rosmadi Ismail ${ }^{2}$
}

Received: 15 July 2021 / Revised: 20 November 2021 / Accepted: 26 November 2021 / Published online: 6 January 2022

(c) The Author(s), under exclusive licence to Korean Society of Nuclear Medicine 2022

\begin{abstract}
Purpose To evaluate the clinical outcome of Q-SPECT/CT in pulmonary thromboembolic disease.

Methods From Jan 2020 to Jan 2021, 30 consecutive patients (M:F=8:22; median age $=52$ year (21-89)) suspected of having acute pulmonary embolism (PE) or chronic thromboembolic pulmonary hypertension (CTEPH) were referred for non-contrasted Q-SPECT/CT. All patients were COVID-19 PCR negative. MSKCC Q-SPECT/CT and/or PISAPED criteria were used to determine the presence of thromboembolic disease in Q-SPECT/CT. Final diagnosis was made based on composite reference standards that included at least 2-month clinical cardiorespiratory assessment and follow-up imaging. Results Q-SPECT/CT was positive in 19 patients: indeterminate in 1 and 10 were negative. Three false positive cases were observed during follow-up. Of the remaining 16 true positives, all patients' cardiorespiratory symptom were improved or stabilised after treatment with anticoagulants. The overall sensitivity, specificity, PPV, NPV and accuracy of Q-SPECT/CT were $100 \%$ (95\% CI, 79.41-100\%), 78.57\% (95\% CI, 49.20-95.34\%), 84.21\% (95\% CI, 66.41-93.57\%), 100\% and 90.00\% (95\% CI, 73.47-97.89\%) respectively.

Conclusions In the current COVID-19 pandemic, Q-SPECT/CT can be an alternative modality to detect pulmonary thromboembolic disease. Normal Q-SPECT/CT excludes pulmonary thromboembolic disease with high degree of certainty. However, false positive has been observed.
\end{abstract}

Keywords Perfusion $\cdot$ SPECT/CT $\cdot$ Thromboembolic $\cdot$ Pulmonary embolism $\cdot$ Chronic thromboembolic pulmonary hypertension

\section{Introduction}

Acute pulmonary embolism (PE) is a life-threatening but treatable illness caused by migration of thrombi to the pulmonary circulation. The mortality rate of acute PE can be as high as $30 \%$ in untreated patients [1,2]. Moreover, chronic PE may organise and increase pulmonary vascular resistance, subsequently leading to chronic thromboembolic pulmonary hypertension (CTEPH) [3, 4].

Teik Hin Tan

teikhin.tan@gmail.com

1 Nuclear Medicine, Sunway Medical Centre, 5, Jalan Lagoon Selatan, Bandar Sunway, 47500 Petaling Jaya, Selangor, Malaysia

2 Internal Medicine, Sunway Medical Centre, 5, Jalan Lagoon Selatan, Bandar Sunway, 47500 Petaling Jaya, Selangor, Malaysia
Imaging plays a pivotal role in the diagnosis and management of these patients. Computed tomography pulmonary angiography (CTPA) is commonly used in the workup of suspected acute PE due to readily availability and rapid acquisition time $[5,6]$.

Ventilation/perfusion single-photon emission computed tomography (V/Q-SPECT) is the second most commonly used modality. V/Q-SPECT has been recently revisited as a standard imaging in evaluating suspected acute PE in young women due to its significantly lower radiation dose to the breast $[7,8]$. Moreover, there is almost no contraindication to undergo V/Q-imaging [6].

For acute PE, V/Q scintigraphy has demonstrated strong diagnostic validations in the prospective outcome studies [6]. In the 2019 ECR guidelines, further confirmatory testing is unnecessary when the VQ scintigraphy is normal or shows high probability of PE [6]. For the evaluation of patients with unexplained pulmonary hypertension and CTEPH, V/Q-SPECT is recommended as the procedure of choice [9]. 
In order to reduce the infectious exposure risk to health care workers in the current COVID-19 pandemics, most nuclear medicine centres have omitted the ventilation component of the V/Q SPECT and substituted it with low-dose CT (LDCT) [10, 11]. Several publications have demonstrated high sensitivities of Q-SPECT and Q-SPECT/CT in evaluation of acute PE and CTEPH [12-16]. Nevertheless, there are some concerns about the higher reported false positive rate of Q-SPECT/CT when comparing to V/Q SPECT [17-19]. Furthermore, data on outcome analysis for Q-SPECT/CT is fairly limited.

The aim of this study is to evaluate the clinical outcomes of patients with pulmonary thromboembolic disease that underwent Q-SPECT/CT and further strengthen the applicability of Q-SPECT/CT in settings.

\section{Methods}

\section{Patients}

From Jan 2020 to Jan 2021, patients with suspected acute PE or CTEPH referred for Q-SPECT/CT were recruited.

Pulmonary Embolism Rule-out Criteria (PERC) and revised Geneva criteria were used to assess clinical probability in patients suspected to have acute PE [21, 22]. Patients were suspected to have CTEPH if they had unexplained raised pulmonary artery systolic pressure (PASP) or right ventricular systolic pressure (RVSP) on echocardiography. Following clinical assessment, all patients underwent D-dimer, COVID-19 PCR test, chest X-ray (CXR) and/or high-resolution CT (HRCT). Some patients additionally underwent Doppler ultrasound of lower limb veins and CTPA.

Patients were then evaluated by Q-SPECT/CT. Exclusion criteria were (1) haemodynamically instability, (2) confirmed PE on CTPA, (3) extensive lung parenchymal abnormality on initial CXR and/or HRCT or (4) positive COVID-19 PCR test.

Following Q-SPECT/CT, patients were managed accordingly by the primary chest physician based on the Q-SPECT/CT and other clinical parameters. They were then followed up for at least 2 months with respect to cardiorespiratory status with/without repeated Q-SPECT/CT, CTPA or HRCT. Final diagnosis was made based on these clinico-radiological findings. The flow chart is depicted in Fig. 1.

This study was approved by the institutional ethics review committee. All patients' data and images were deidentified and anonymised before being used as inputs for data processing and analysis.

\section{Imaging Acquisition and Reconstruction}

Q-SPECT/CT was performed in accordance with the recommended guidelines and protocols [23]. Patients were administered with $185 \mathrm{MBq}$ of $99 \mathrm{mTc}$-macroaggregated albumin (MAA) (Pulmocis ${ }^{\circledR}$, IBA). Image acquisition was performed within 5 min post-injection on a dedicated Siemens Symbia Intevo 16. Multiple planar projections were acquired followed by SPECT and free-breathing non-contrasted LDCT.

For SPECT acquisition, a general purpose collimator, $128 \times 128$ matrix, was used. A peak window of $15 \%$ width was centred around the $140-\mathrm{keV}$ energy peak. The total number of projections is 32 , at $20 \mathrm{~s}$ per projection. For CT imaging, $130 \mathrm{kV}$ and $75 \mathrm{mAs} / \mathrm{slice}$ were used. Attenuation and scatter correction were performed using CT-based attenuation-corrected maps.

SPECT images were reconstructed with an ordered-subset expectation maximisation (OSEM) 3-D algorithm (4 subsets, 4 iterations, 8.40 Gausian filter). CT transverse images were reconstructed at $1.5-\mathrm{mm}$ section thicknesses, using reconstruction kernels of sharp resolution (Siemens B75f).

\section{Image Interpretation}

All images were viewed on (Syringo.via workstation; Siemens Healthineers) by an experienced nuclear medicine physician. Segmental map was used when interpreting multiplanar images. All three orthogonal planes of the SPECT, CT and fusion images were viewed.

In this study, MSKCC Q-SPECT/CT and/or PISAPED criteria were used to determine the presence of thromboembolic disease in Q-SPECT/CT. By MSKCC Q-SPECT/CT criteria, any wedge-shaped peripheral perfusion defect occupying $>50 \%$ of a lung segment without corresponding pulmonary parenchymal or pleural disease was considered positive [24]. In the PISAPED criteria, single or multiple wedgeshaped perfusion defects of any sizes are considered positive [25]. Perfusion defect that did not fulfil the above criteria was classified as indeterminate.

\section{Statistical Analysis}

The statistical analysis includes sensitivity, specificity, positive predictive value (PPV) and negative predictive value (NPV) calculated using $2 \times 2$ table and MedCalc calculator.

\section{Results}

A total of 30 patients (male:female $=8: 22$; median age $=52$ year (range 21 to 89 years)) with suspicion of acute $\mathrm{PE}$ or CTEPH were included during the study period (Table 1). 
Fig. 1 Flow chart of workout and follow-up of suspected PE and CTEPH patients referred for Q-SPECT/CT

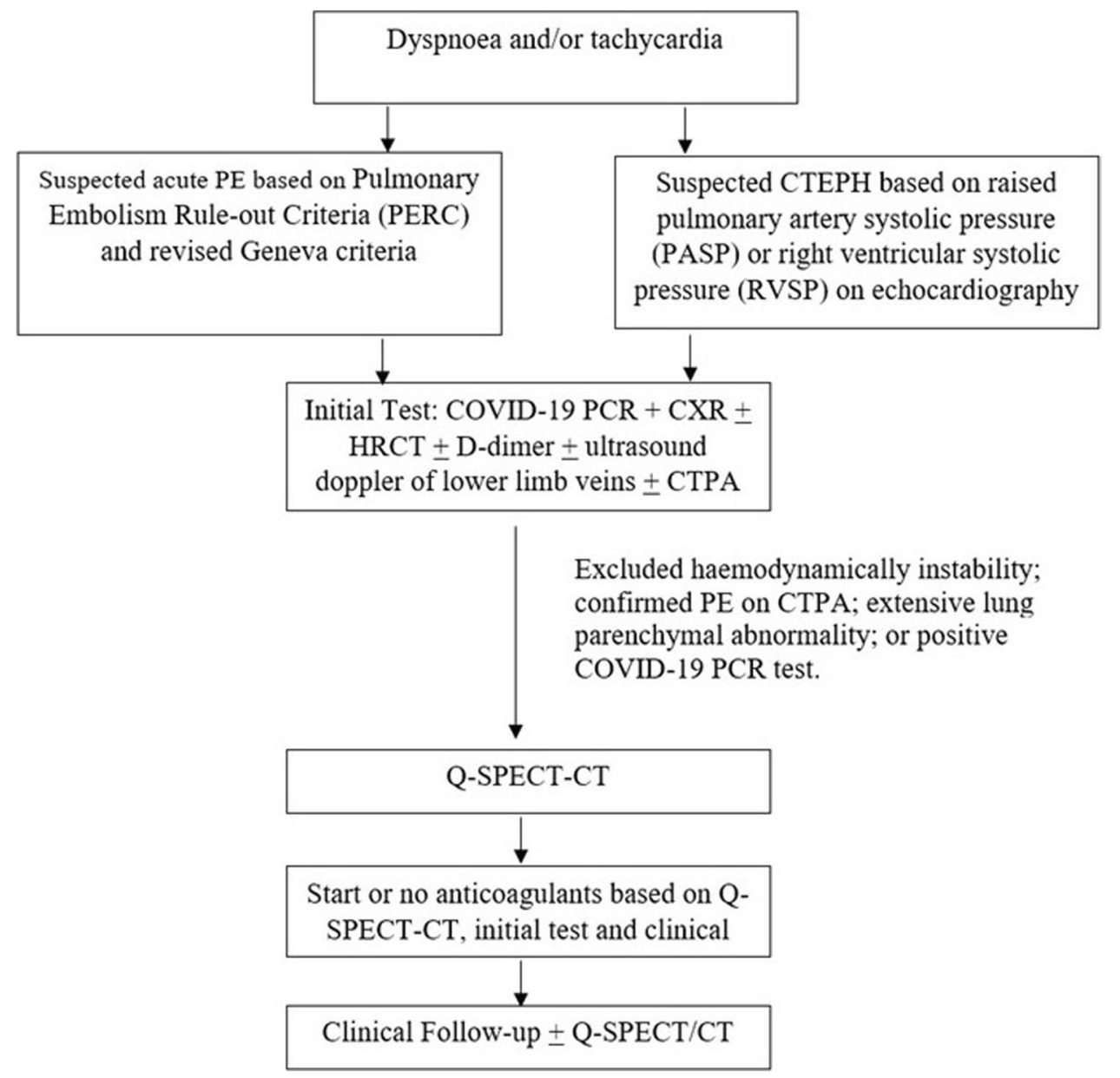

Nineteen out of thirty patients $(63.3 \%)$ primarily presented with sudden-onset or worsened resting dyspnoea. A diagnosis of acute PE was unable to be excluded based on PERC criteria [13]. Of these 19 patients, 8 patients (41.2\%) had predisposing risk factors of PE and classified as intermediate risk following the revised Geneva criteria [14]. Half of the patients $(50.0 \%)$ demonstrated raised D-dimer levels. Seven patients $(36.8 \%)$ had CTPA done, 3 of which revealed inconclusive finding of PE and 4 of which were reported as normal (Table 1).

Eleven patients $(36.7 \%)$ presented with dyspnoea of uncertain origin and demonstrated raised pulmonary artery systolic pressure (PASP) and right ventricular systolic pressure (RVSP) on echocardiography. A diagnosis of CTEPH was suspected in these patients.

\section{Positive Q-SPECT/CT in PE Group}

Out of 19 patients who were suspected to have acute PE, thromboembolic disease was detected by Q-SPECT/CT in 10 patients.
Of the 10 patients, 8 were given anticoagulants. Seven of them demonstrated resolution of tachycardia and improvement in dyspnoea during the 2-month follow-up. Complete or partial resolution of perfusion defect(s) were observed on follow-up Q-SPECT/CT (Fig. 2). Hence, all 7 patients were considered true positive for the diagnosis of acute $\mathrm{PE}$ (Table 2).

The other 3 patients were classified as false positive. In these 3 patients, one patient continued to have dyspnoea despite anticoagulants. Follow-up Q-SPECT/CT showed perfusion defects at hyperexpanded oligaemic lung segments (Fig. 3). She was finally diagnosed with right coronary artery stenosis on subsequent coronary angiography.

The remaining 2 false-positive patients were not treated with anticoagulant. On follow-up, their cardiorespiratory symptom resolved without specific therapy. No follow-up Q-SPECT/CT was performed as it was deemed unnecessary by the chest physician. One patient was eventually diagnosed with cardiac failure. Retrospective review of the Q-SPECT/ CT images revealed upper lobe diversion of 99mTc-MAA with presence of lower lobar perfusion defects (Fig. 3). The 
Table 1 Baseline distribution of sociodemographic and subjects' characteristics

\begin{tabular}{lll}
\hline Characteristic, total patients $=\mathbf{3 0}$ & & Number, $\boldsymbol{n}(\boldsymbol{\%})$ \\
\hline Gender & Female & $22(73.3 \%)$ \\
Age in year, median (range) & Male & $8(26.6 \%)$ \\
Clinical impression & Suspected PE & $52(21-89)$ \\
& Suspected CTEPH & $19(63.3 \%)$ \\
PE group, Total patients $=\mathbf{1 9}$ & & $11(36.6 \%)$ \\
Predisposing factors PE & Previous PE & \\
& Active cancer & $1(5.6 \%)$ \\
& Immobility due to long flight & $2(10.5 \%)$ \\
& Pneumonia & $1(5.6 \%)$ \\
Clinical probability & Low & $4(22.2 \%)$ \\
& Intermediate & $10(52.6 \%)$ \\
Other tests & High & $8(42.1 \%)$ \\
& Raised D-dimer & $1(5.3 \%)$ \\
& Suspicious/normal CTPA & $7(36.8 \%)$ \\
& Raised D-dimer + suspicious CTPA & $4(21.1 \%)$ \\
& Normal US Doppler lower limb & $3(16.7 \%)$ \\
& Positive DVT & $8(42.1 \%)$ \\
Echocardiography & Low/ desaturation oxygenation & $1(5.3 \%)$ \\
Other tests & & $3(15.8 \%)$ \\
& Raised PASP or RVSP* & \\
\hline & Suspicious/normal CTPA & $11(100 \%)$ \\
& Normal US Doppler lower limb & $6(54.5 \%)$ \\
& Positive deep vein thrombosis on US & $2(18.2 \%)$ \\
& & $1(9.1 \%)$ \\
\hline
\end{tabular}

${ }^{*} P A S P$ pulmonary artery systolic pressure, RVSP right ventricular systolic pressure
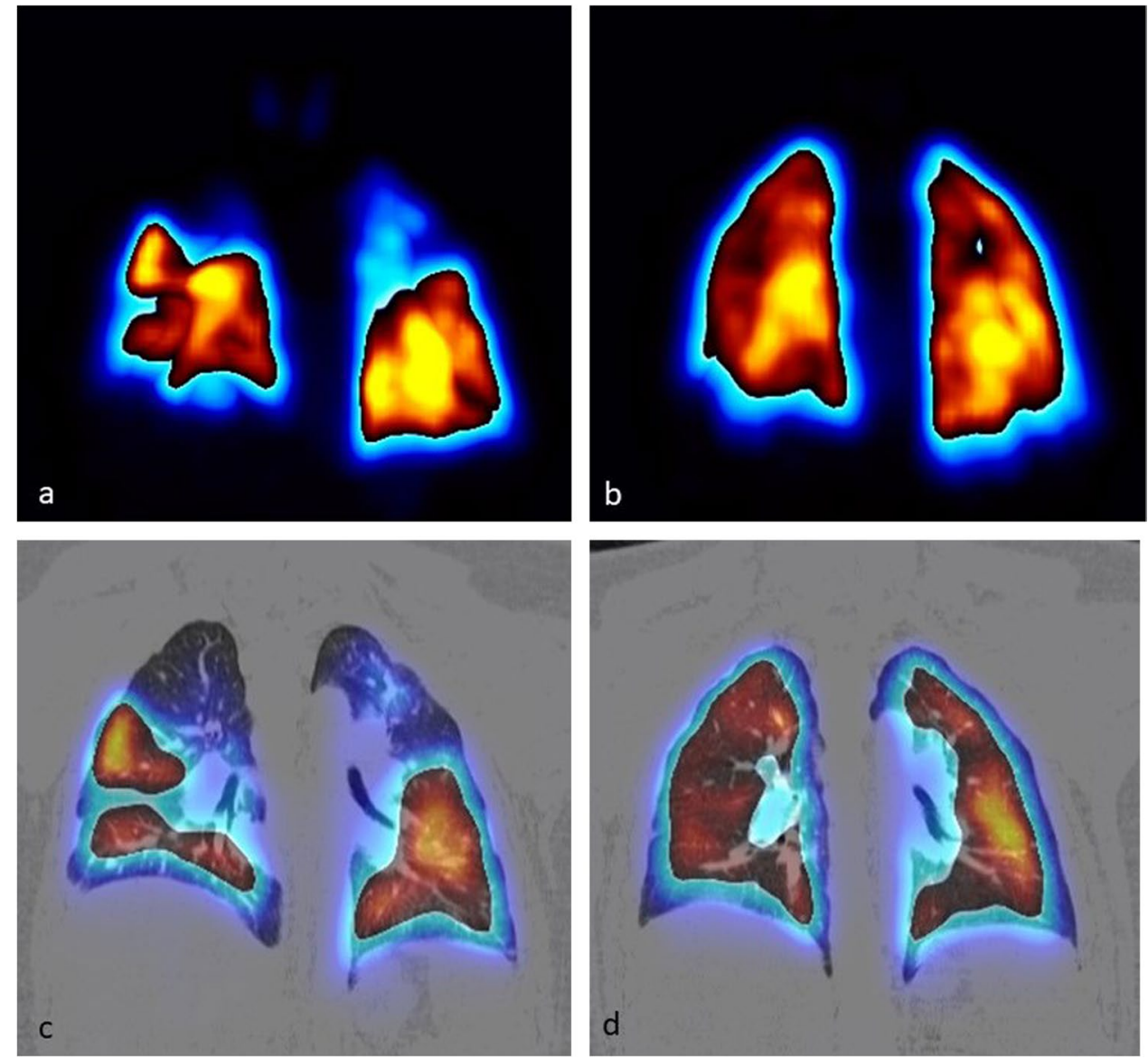

Fig. 2 A 21-year-old lady presented with sudden onset of resting dyspnoea. She had history of 12-h long hauled flight. Initial ultrasound Doppler low limbs and chest X-ray were normal. Q-SPECT/CT showed multiple large and moderate segmental perfusion defects consistent with acute pulmonary embolism (a, c). After receiving apixaban, follow-up Q-SPECT/ CT showed remarkable resolution of PE (b, d) 
Table 2 Outcome of suspected PE and CTEPH patients after Q-SPECT/CT

\begin{tabular}{|c|c|c|c|c|}
\hline & \multirow[t]{2}{*}{ Q-SPECT/CT results } & & Outcome after 2 months follow-up & \multirow[b]{2}{*}{ Total } \\
\hline & & Positive & Negative & \\
\hline \multirow[t]{2}{*}{ PE group, $n=19$} & Positive & 7 & 3 & 10 \\
\hline & Negative & 0 & $9 *$ & 9 \\
\hline \multirow[t]{3}{*}{ CTEPH group, $n=11$} & Positive & 9 & 0 & 9 \\
\hline & Negative & 0 & 2 & 2 \\
\hline & Total & 16 & 14 & 30 \\
\hline \multicolumn{5}{|c|}{ *Including 1 patient reported as indeterminate } \\
\hline \multicolumn{2}{|c|}{$\begin{array}{l}\text { Overall Q-SPECT characteristics } \\
\text { Sensitivity } \\
\text { Specificity } \\
\text { PPV } \\
\text { NPV } \\
\text { Accuracy }\end{array}$} & \multicolumn{2}{|l|}{$\begin{array}{l}=100 \%(95 \% \text { CI, } 79.41-100 \%) \\
=78.57 \%(95 \% \text { CI, } 49.20-95.34 \%) \\
=84.21 \%(95 \% \text { CI, } 66.17-93.57 \%) \\
=100 \% \\
=90.00 \%(95 \% \text { CI, } 73.47-97.89 \%)\end{array}$} & \\
\hline
\end{tabular}

second patients' diagnosis was unknown and retrospective CT review did not reveal obvious morphological changes.

\section{Positive Q-SPECT/CT in CTEPH Group}

Out of 11 patients who were suspected to have CTEPH, thromboembolic disease was detected by Q-SPECT/CT in 9 patients.

All 9 patients received anticoagulants. Five patients demonstrated improved cardiorespiratory symptom during clinical follow-up, whereas 4 patients' status remained stable.

As CTEPH patients on long-term anticoagulants are expected to have no further in situ pulmonary artery thrombosis and recurrent venous thromboembolism [26], these patients were classified as true positive based on clinical disease stabilisation (Table 2).

\section{Negative Thromboembolic Disease by Q-SPECT/CT}

Nine patients from suspected acute PE group and 2 patients from suspected CTEPH group demonstrated either negative or indeterminate finding of thromboembolic disease on Q-SPECT/CT (Table 2).

Out of these 9 patients from acute PE group, 8 patients demonstrated low pre-test clinical probability for thromboembolic disease and were not treated with anticoagulants. Their cardiorespiratory conditions had not deteriorated after 2 months of follow-up. One patient had history of ovarian carcinoma with positive D-dimer and deep vein thrombosis (DVT) on ultrasound. Anticoagulants were prescribed to treat the DVT. The diagnosis of absent PE was confirmed by negative pre- and posttreatment CTPA.

In 2 patients from CTEPH group, no anticoagulant was prescribed. Their cardiorespiratory conditions did not deteriorate after 2 months of follow-up.
Hence, all 11 patients with negative or indeterminate Q-SPECT/CT were classified as true negatives.

The overall sensitivity, specificity, PPV, NPV and accuracy of Q-SPECT/CT were $100 \%$ (95\% confidence interval (CI), 79.41-100\%), 78.57\% (95\% CI, 49.20-95.34\%), $84.21 \%$ (95\% CI, 66.17-93.57\%), 100\% and $90.00 \%(95 \%$ CI, 73.47-97.89\%) respectively (Table 2).

\section{Discussion}

Extensive studies have demonstrated high sensitivity, specificity and NPV of V/Q-SPECT in the evaluation of acute PE, ranging between $96-99 \%, 96-98 \%$ and $97-99 \%$ respectively [27-32].

When CT is added in V/Q SPECT, the specificity increased to $100 \%$ and lower non-diagnostic rate is observed [15]. A recent analysis by Toney et al. has demonstrated that V/Q SPECT/CT confers better economic value when compared to CTPA, V/Q SPECT and V/Q planar imaging, primarily via improved sensitivity and specificity and lower nondiagnostic rates [33].

In the diagnosis of CTEPH, V/Q planar imaging and Q-SPECT are reported to have higher sensitivities and equivalent specificities compare to CTPA (96-97\% vs 51\% and $90-95 \%$ vs $99 \%$ respectively) $[14,34]$.

Compared to V/Q-SPECT, Q-SPECT/CT has comparable sensitivity but is reported to have higher false positive rate in PE evaluation $[17,19,20]$. However, such a high false positive rate is not observed in CTEPH [9].

At our institution, appropriate use criteria of PE imaging endorsed by the Society of Nuclear Medicine and Molecular Imaging (SNMMI), the European Association of Nuclear Medicine (EANM), the American Society of Hematology (ASH), the Society of Thoracic Surgeons (STS) and the 

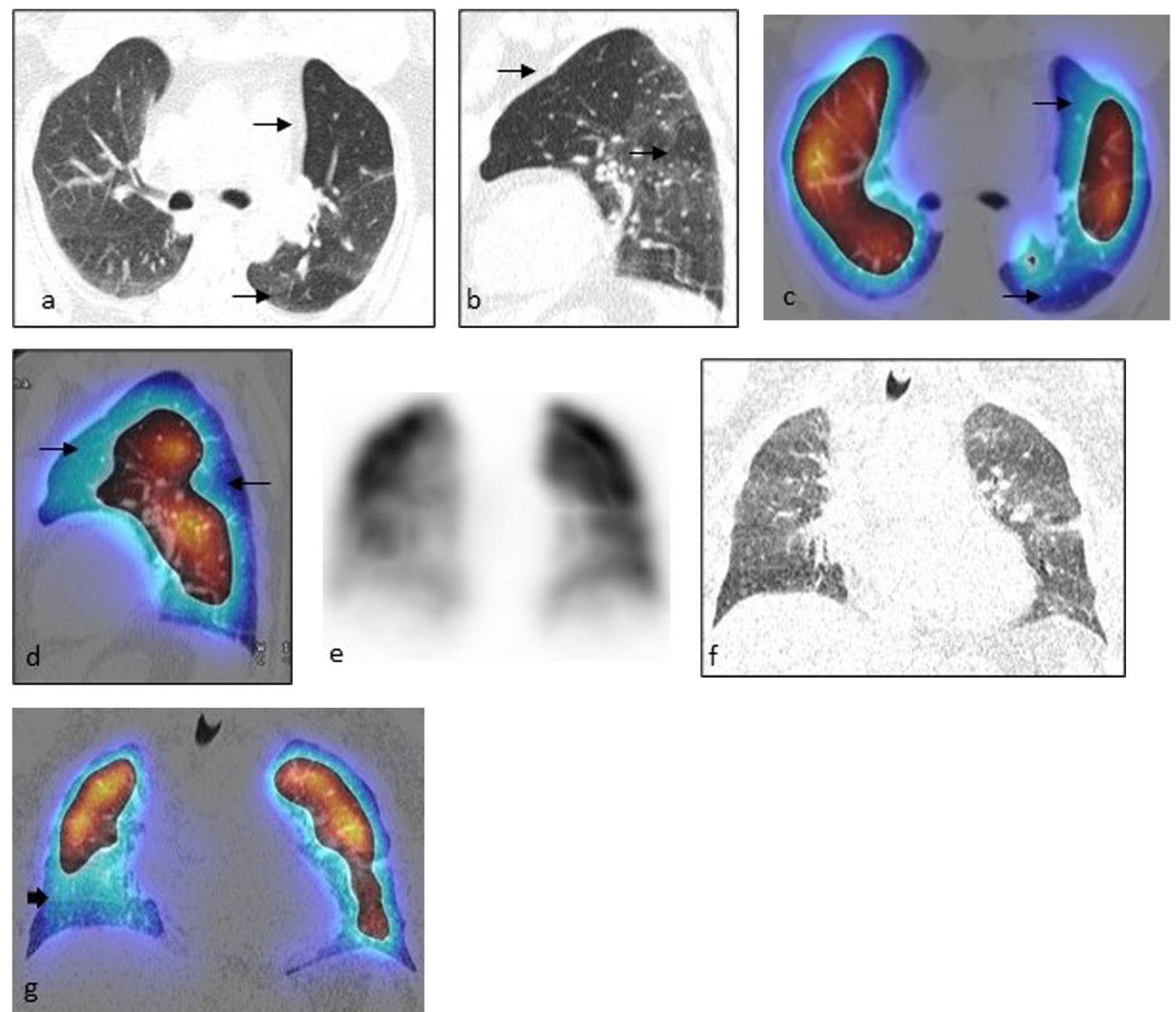

Fig. 3 Two false positive cases. The first case showed large and moderate segmental perfusion defects which corresponded to hyperexpanded oligaemic lung parenchyma on Q-SPECT/CT (arrow, a-d).

American College of Emergency Physicians (ACEP) were followed [35].

CTPA remains the primary form of imaging for evaluation of patients with high pre-test probability of acute $\mathrm{PE}$, particularly those with haemodynamic instability. When there is equivocal or suspected small PE on CTPA, Q-SPECT/CT is performed to exclude it. In the case of low or intermediate pre-test probability, Q-SPECT/CT may be used as initial workup imaging in view of high NPV and lower radiation dose, in particular to female breasts [7, 8]. The absence of contra-indications to Q-SPECT/CT also makes it a preferred option for patients with kidney impairment [6].
The second case was diagnosed as cardiac failure. Q-SPECT/CT showed upper lobe diversion of 99mTc-MAA resulting lower lobar perfusion defects. Cardiomegaly was also seen (bold arrow, $\mathbf{e}-\mathbf{g}$ )

A significant number of venous and pulmonary thromboembolic complications have been observed in COVID19 patients, especially critically ill patients [36, 37]. Those patients were excluded in our study because of the high likelihood of lung parenchymal abnormality that may lead to difficulties of interpretation and potential false positive results [38]. Moreover, the pathogenesis and management of thromboembolic events in COVID-19 were not fully understood during the time of data collection and may have potentially confounded the subsequent outcome analysis.

Based on our findings, Q-SPECT/CT demonstrates $100 \%$ sensitivity and $100 \%$ NPV. Such high sensitivity is inconsistent with previous numerous studies by Mazurek 
et al., Sevda et al. and Wang et al., where the sensitivity ranges between 93 and 100\% [12, 13, 16]. Furthermore, high NPV implies that a normal Q-SPECT/CT is able to exclude PE or CTEPH with high degree of confidence. In our study, no worsening of clinical outcome was observed when Q-SPECT/CT was normal.

Three patients were falsely positive in our study, of which one patient showed segmental hyperexpanded oligaemic lung parenchyma with perfusion defects on Q-SPECT/CT. Although perfusion defect in emphysema is uncommon, such findings have been reported and are postulated to be related to vascular changes [39, 40]. Cardiac failure can result in upper lobe diversion of $99 \mathrm{mTc}-\mathrm{MAA}$ which may lead to lower lobar perfusion defects mimicking embolic defects. Therefore, we suggest that familiarity with features of emphysema on $\mathrm{CT}$ and clinical correlation may minimise such potential pitfalls [41, 42].

Our observed false positive rate was higher as compare to previous studies conducted by Le Roux et al., Palmowski et al. and Wang et al. (21.4\% versus $14.8 \%, 17.3 \%$ and $9.4 \%$ respectively) $[16,19,20]$. The possible reason of higher false positive rate observed in our study is partly due to the use of different reference standards. Both Le Roux's and Palmowski's studies used VQ-SPECT and Wang's study used pulmonary angiography as reference standards, whereas clinical endpoint was used in our study as reference standard.

The limitation of this study is small sample size, which is partly due to low number of referrals during COVID-19 pandemics, exclusion of CTPA confirmed case and exclusion of patients with obvious lung parenchymal disease on initial CXR and HRCT. Another limitation is absence of control group for comparison.

Another potential pitfall is the classification of true negative when there is absence of clinical deterioration over 2-month observation period. Although a 2-month cutoff period was used for the purpose of outcome analysis, it is possible that asymptomatic microthrombi went undetected and were therefore missed during the observation period.

In conclusion, our outcome analysis suggests that Q-SPECT/CT has high sensitivity and NPV in evaluating pulmonary thromboembolic disease. Normal Q-SPECT/ $\mathrm{CT}$ excludes pulmonary thromboembolic disease with high degree of certainty. We suggest no further testing when the $\mathrm{Q}-\mathrm{SPECT} / \mathrm{CT}$ is normal.

However, false positive has been observed and careful clinical and CT correlation may be warranted in such and additional testing may be necessary as recommended by Zuckier et al. [10].

In the current COVID-19 pandemics, Q-SPECT/CT can be an alternative modality to detect pulmonary thromboembolic disease in view of the exposure risks associated with ventilation study.
Acknowledgements The authors would like to thank Dr. Jay Suriar Rajasuriar, Dr. Usha Rani George, Dr. Lai-Lan Yoon and all the personnel in Nuclear Medicine Centre and Sunway Medical Centre for their assistance in this study.

Author Contribution Teik Hin Tan: conceptualisation and design, investigation, analysis of data, drafting and approva of the manuscript. Rosmadi Ismail: data acquisition, review of writing, final approval.

Data availability Please contact author for data requests.

\section{Declarations}

Ethical Approval and Consent for Participation All procedures performed in studies were in accordance with the ethical standards of the institution and with the 1964 Helsinki declaration and its later amendments or comparable ethical standards. The institution review board approved this retrospective study, and the requirement to obtain informed consent was waived.

Consent for Publication The institution review board approved this retrospective study for publication.

Conflict of Interest Teik-Hin Tan and Rosmadi Ismail declare no conflict of interest.

\section{References}

1. Bĕlohlávek J, Dytrych V, Linhart A. Pulmonary embolism, part I: epidemiology, risk factors and risk stratification, pathophysiology, clinical presentation, diagnosis and nonthrombotic pulmonary embolism. Exp Clin Cardiol. 2013;18:129-38.

2. Goldhaber SZ, Visani L, De Rosa M. Acute pulmonary embolism: clinical outcomes in the International Cooperative Pulmonary Embolism Registry (ICOPER). Lancet. 1999;353:1386-9.

3. Lang I. Chronic thromboembolic pulmonary hypertension:a distinct disease entity. Eur Respir Rev. 2015;24:246-52.

4. Fedullo P, Kerr KM, Kim NH, Auger WR. Chronic thromboembolic pulmonary hypertension. Am J Respir Crit Care Med. 2011;183:1605-13.

5. Moore AJE, Wachsmann J, Chamarthy MR, Panjikaran L, Tanabe Y, Rajiah P. Imaging of acute pulmonary embolism: an update. Cardiovasc Diagn Ther. 2018;8:225-43.

6. Konstantinides SV, Meyer G, Becattini C, Bueno H, Geersing GJ, Harjola VP, et al. 2019 ESC Guidelines for the diagnosis and management of acute pulmonary embolism developed in collaboration with the European Respiratory Society (ERS). Eur Heart J. 2020;41:543-603.

7. Hansen SL, de Nijs R, Mortensen J, Berg RMG. Ventilation-perfusion SPECT versus CTPA in young adult females with suspected pulmonary embolism. Eur Respir J. 2020;55:2000448.

8. Astani SA, Davis LC, Harkness BA, Supanich MP, Dalal I. Detection of pulmonary embolism during pregnancy: comparing radiation doses of CTPA and pulomonary scintigraphy. Nucl Med Commun. 2014;35(704):11.

9. Moradi F, Morris TA, Hoh CK. Perfusion scintigraphy in diagnosis and management of thromboembolic pulmonary hypertension. Radiographics. 2019;39:169-85.

10. Zuckier LS, Moadel RM, Haramati LB, Freeman LM. Diagnostic evaluation of pulmonary embolism during the COVID-19 pandemic. J Nucl Med. 2020;61:630-1. 
11. Lu Y, Macapinlac HA. Perfusion SPECT/CT to diagnose pulmonary embolism during COVID-19 pandemic. Eur J Nucl Med Mol Imaging. 2020;47:2064-5.

12. Mazurek A, Dziuk M, Witkowska-Patena E, Piszczek S, Gizewska A. The utility of hybrid SPECT/CT lung perfusion scintigraphy in pulmonary embolism diagnosis. Respiration. 2015;90:393-401.

13. Sevda Naydenska, Dora Zlatareva, Bilyana Stancheva, Elina Smilkova, Radoslav Bilyukov, Pencheva $\mathrm{V}$, et al. Diagnostic value of perfusion lung scintigraphy in SPECT/CT and computed tomography pulmonary angiography for detection of pulmonary embolism. Eur Respir J. 2017;50:61PA2358.

14. Soler X, Kerr KM, Marsh JJ, Renner JW, Hoh CK, Test VJ, et al. Pilot study comparing SPECT perfusion scintigraphy with CT pulmonary angiography in chronic thromboembolic pulmonary hypertension. Respirology. 2012;17:180-4.

15. Gutte H, Mortensen J, Jensen CV, Johnbeck CB, von der Recke $\mathrm{P}$, Petersen CL, et al. Detection of pulmonary embolism with combined ventilation-perfusion SPECT and low-dose CT: headto-head comparison with multidetector CT angiography. J Nucl Med. 2009;50:1987-92.

16. Wang L, Wang M, Yang T, Wu D, Xiong C, Fang W. A prospective, comparative study of ventilation-perfusion planar imaging and ventilation-perfusion SPECT for chronic thromboembolic pulmonary hypertension. J Nucl Med. 2020;61:1832-8.

17. Thanuja M, Maimanah M, Sara U. Diagnosis of pulmonary embolism: a comparison between ventilation/perfusion SPECT/CT and perfusion-only SPECT/CT. Med J Malaysia. 2020;75:490-3.

18. Le Roux PY, Le Gal G, Salaun PY. Lung scintigraphy for pulmonary embolism diagnosis during the COVID-19 pandemic: does the benefit-risk ratio really justify omitting the ventilation study? Eur J Nucl Med Mol Imaging. 2020;47:2499-500.

19. Le Roux PY, Robin P, DellucA, Abgral R, Palard X, Tissot V, et al. Additional value of combining low-dose computed tomography to V/Q SPECT on a hybrid SPECT-CT camera for pulmonary embolism diagnosis. Nucl Med Commun. 2015;36:922-30.

20. Palmowski K, Oltmanns U, Kreuter M, Mottaghy FM, Palmowski M, Behrendt FF. Diagnosis of pulmonary embolism: conventional ventilation/perfusion SPECT is superior to the combination of perfusion SPECT and nonenhanced CT. Respiration. 2014;88:291-7.

21. Kline JA, Mitchell AM, Kabrhel C, Richman PB, Courtney DM. Clinical criteria to prevent unnecessary diagnostic testing in emergency department patients with suspected pulmonary embolism. J Thromb Haemost. 2004;2:1247-55.

22. Le Gal G, Righini M, Roy PM, Sanchez O, Aujesky D, Bounameaux $\mathrm{H}$, et al. Prediction of pulmonary embolism in the emergency department: the revised Geneva score. Ann Intern Med. 2006;144:165-71.

23. Bajc M, Schümichen C, Grüning T, Lindqvist A, Le Roux PY, Alatri A, et al. EANM guideline for ventilation/perfusion singlephoton emission computed tomography (SPECT) for diagnosis of pulmonary embolism and beyond. Eur J Nucl Med Mol Imaging. 2019;46:2429-51.

24. Lu Y, Lorenzoni A, Fox JJ, Rademaker J, Vander Els N, Grewal RK, et al. Noncontrast perfusion single-photon emission CT/CT scanning: a new test for the expedited, high-accuracy diagnosis of acute pulmonary embolism. Chest. 2014;145:1079-88.

25. Miniati M, Pistolesi M, Marini C, Di Ricco G, Formichi B, Prediletto $\mathrm{R}$, et al. Value of perfusion lung scan in the diagnosis of pulmonary embolism: results of the Prospective Investigative Study of Acute Pulmonary Embolism Diagnosis (PISA-PED). Am J Respir Crit Care Med. 1996;154:1387-93.

26. Pepke-Zaba J, Jansa P, Kim NH, Naeije R, Simonneau G. Chronic thromboembolic pulmonary hypertension: role of medical therapy. Eur Respir J. 2013;41:985-90.
27. Bajc M, Olsson B, Palmer J, Jonson B. Ventilation/perfusion SPECT for diagnostics of pulmonary embolism in clinical practice. J Intern Med. 2008;264:379-87.

28. Grüning T, Drake BE, Farrell SL, Nokes T. Three-year clinical experience with VQ SPECT for diagnosing pulmonary embolism: diagnostic performance. Clin Imaging. 2014;38:831-5.

29. Corbus HF, Seitz JP, Larson RK, Stobbe DE, Wooten W, Sayre JW, et al. Diagnostic usefulness of lung SPET in pulmonary thromboembolism: an outcome study. Nucl Med Commun. 1997;18:897-906.

30. Le Duc-PennecA, Le Roux PY, Cornily JC, Jaffrelot M, Delluc A, de Saint-Martin L, et al. Diagnostic accuracy of single-photon emission tomography ventilation/perfusion lung scan in the diagnosis of pulmonary embolism. Chest. 2012;141:381-7.

31. Kan Y, Yuan L, Meeks JK, Li C, Liu W, Yang J. The accuracy of V/Q SPECT in the diagnosis of pulmonary embolism: a metaanalysis. Acta Radiol. 2015;56:565-72.

32. Patel P, Patel P, Bhatt M, Braun C, Begum H, Wiercioch W, et al. Systematic review and meta-analysis of test accuracy for the diagnosis of suspected pulmonary embolism. Blood Adv. 2020;4:4296-311.

33. Toney LK, Kim RD, Palli SR. The economic value of hybrid single-photon emission computed tomography with computed tomography imaging in pulmonary embolism diagnosis. Acad Emerg Med. 2017;24:1110-23.

34. Tunariu N, Gibbs SJ, Win Z, Gin-Sing W, Graham A, Gishen P, et al. Ventilation-perfusion scintigraphy is more sensitive than multidetector CTPA in detecting chronic thromboembolic pulmonary disease as a treatable cause of pulmonary hypertension. J Nucl Med. 2007;48:680-4.

35. Waxman AD, Bajc M, Brown M, Fahey FH, Freeman LM, Haramati LB, et al. Appropriate use criteria for ventilation-perfusion imaging in pulmonary embolism: summary and excerpts. J Nucl Med. 2017;58:13N-15N.

36. Mondal S, Quintili AL, Karamchandani K, Bose S. Thromboembolic disease in COVID-19 patients: a brief narrative review. $\mathrm{J}$ intensive care. 2020;8:70.

37. Suh YJ, Hong H, Ohana M, Bompard F, Revel MP, Valle $\mathrm{C}$, et al. Pulmonary embolism and deep vein thrombosis in COVID-19: a systematic review and meta-analysis. Radiology. 2021;298:E70-80.

38. Cobes N, Guernou M, Lussato D, Queneau M, Songy B, Bonardel $\mathrm{G}$, et al. Ventilation/perfusion SPECT/CT findings in different lung lesions associated with COVID-19: a case series. Eur J Nucl Med Mol Imaging. 2020;47:2453-60.

39. Jögi J, Markstad H, Tufvesson E, Bjermer L, Bajc M. The added value of hybrid ventilation/perfusion SPECT/CT in patients with stable COPD or apparently healthy smokers. Cancer-suspected CT findings in the lungs are common when hybrid imaging is used. Int J Chron Obstruct Pulmon Dis. 2014;10:25-30.

40. Bajc M, Chen Y, Wang J, Li XY, Shen WM, Wang CZ, et al. Identifying the heterogeneity of COPD by V/P SPECT: a new tool for improving the diagnosis of parenchymal defects and grading the severity of small airways disease. Int J Chron Obstruct Pulmon Dis. 2017;12:1579-87.

41. Lynch DA, Austin JH, Hogg JC, Grenier PA, Kauczor HU, Bankier AA, et al. CT-definable subtypes of chronic obstructive pulmonary disease: a statement of the Fleischner Society. Radiology. 2015;277:192-205.

42. Schembri GP, Roach PJ, Bailey DL, Freeman L. Artifacts and anatomical variants affecting ventilation and perfusion lung imaging. Semin Nucl Med. 2015;45:373-91.

Publisher's Note Springer Nature remains neutral with regard to jurisdictional claims in published maps and institutional affiliations. 